\title{
Genetics of carbohydrate metabolism and growth in Eisenia foetida (Oligochaeta: Lumbricidae)
}

\author{
Walter J. Diehl
}

\begin{abstract}
Department of Biological Sciences, Mississippi State University, P.O. Drawer GY, Mississippi State, MS 39762.
\end{abstract}

The heterozygosity of 13 polymorphic loci, encoding 10 enzymes from several pathways in carbohydrate metabolism, was tested for its effect on growth in juvenile manure worms Eisenia foetida $(n=169)$, raised under stressful (limited food, low moisture) followed by non-stressful (abundant food, high moisture) conditions. The predictive value of heterozygosity on growth was greatly improved by treating each locus separately rather than summing heterozygosity across all loci (multilocus heterozygosity). Under non-stressful conditions, heterozygosity among loci treated separately had no effect on growth rate $(F=1.60 ; \mathrm{df}=13,155 ; \mathrm{NS})$; whereas under stressful conditions, heterozygosity among loci treated separately had significant and differential effects on growth rate $(F=2.34 ; \mathrm{df}=13,155 ; P<0.01)$ with 7 loci contributing to a positive heterozygosity-growth rate correlation $(r=0 \cdot 235 ; P<0 \cdot 005)$ and 6 loci contributing to a negative heterozygosity-growth rate correlation $(r=-0 \cdot 202, P<0 \cdot 01)$. Implicated loci could not be easily grouped into specific metabolic pathways. The negative contribution by some loci may arise as a consequence of heterozygote inferiority, linkage disequilibria with positively-contributing loci, or strong selection at that locus.

\section{INTRODUCTION}

Multilocus heterozygosity, measured cumulatively for a small number of electrophoretically detectable loci, has been correlated with fitness-related parameters in a variety of experimental organisms (see reviews by Mitton and Grant, 1984; Zouros and Foltz, 1987). Briefly, multilocus heterozygosity is often positively correlated with age-specific growth, survivorship, and fecundity in large panmictic populations. The physiological basis for this phenomenon is postulated to be economy of standard metabolism arising from efficiency of protein synthesis and turnover (Koehn and Shumway, 1982; Hawkins et al., 1986).

The effects of multilocus heterozygosity on fitness-related parameters may be exaggerated, thus more easily visualized, under conditions of environmental stress. Koehn and Shumway (1982) showed that metabolic costs, measured as weightspecific oxygen consumption, increased disproportionately in homozygous Crassostrea virginica compared with heterozygous individuals when stressed by both high temperature and low salinity. Rodhouse and Gaffney (1984) showed that heterozygous $C$. virginica lost weight during starva- tion more slowly than homozygous individuals. Diehl et al. (1986) showed that reduced metabolic costs in heterozygous Mytilus edulis were associated more with resistance to weight loss than with capacity for weight gain. Thus the advantages conferred by heterozygosity appear to be of primary benefit when the environment challenges the energy balance of the individual. In $M$. edulis this may be manifest as improved survivorship of more heterozygous individuals during the energeticallystressful fall and winter months (Diehl and Koehn, 1985).

Until recently, the contributions of single loci to the heterozygosity-growth phenomenon were thought to be uniformly small, often undetectable, a conclusion resulting from the numerous studies employing limited sample sizes of both individuals and polymorphic loci (e.g., Koehn and Gaffney, 1984). It is now obvious from studies incorporating relatively large numbers of individuals and loci that single-locus effects are, in fact, not uniform (Bush et al., 1987; Koehn et al., 1988). Further, differential contributions by loci argue forcefully for a causal role in determining growth rate (Bush et al., 1987), particularly if "important" loci can be grouped into specific metabolic pathways which 
provide energy or raw materials for growth. Koehn et al. (1988) implicated enzymes catalyzing reactions of protein catabolism, preglycolysis and glycolysis as contributing more to the multilocus heterozygosity-growth relationship in Mulinia lateralis than enzymes involved in other pathways (e.g., pentose shunt, detoxification). Rates of preglycolytic and glycolytic reactions may potentially impact a variety of energetic conditions of an individual including ATP levels and energy charge, redox balance, glycogen synthesis and utilization.

The present experiment was undertaken to determine the contribution of heterozygosity among loci encoding enzymes of carbohydrate metabolism to growth in the manure worm Eisenia foetida (Oligochaeta: Lumbricidae) cultured under stressful vs. non-stressful conditions. To the extent examined, lumbricid earthworms possess a full complement of glycolytic, pentose shunt and citric acid cycle enzymes (Dastolli, 1964). Thus far, polymorphisms have been identified for only a few of the loci encoding these enzymes in $E$. foetida (Jaenike, 1982; Robotti, 1982). In the current experiment however, 13 polymorphic loci encoding ten enzymes are identified from a variety of relevant pathways including preglycolysis, glycolysis, pentose shunt, citric acid cycle, redox balance, and glycerol synthesis. Thus in the study of the heterozygosity-growth phenomenon, this experiment represents the first attempt to evaluate loci encoding enzymes from a unified set of interrelated metabolic pathways.

\section{METHODS AND MATERIALS}

Approximately 750 E. foetida were purchased from a commercial bait farm in West Point, Mississippi, U.S.A. Individuals were taken from concrete bunkers filled with damp peat moss (surface area $=$ $2.2 \mathrm{~m}^{2}$; depth $=0.4 \mathrm{~m}$ ); each bunker was estimated to contain 40,000 large, adult individuals plus numerous but uncounted small adults and juveniles. These earthworms were originally derived from a mixture of commercial stocks in Mississippi and Texas and have been maintained locally for six years.

In the laboratory, juveniles were separated from adults and were raised singly in $200 \mathrm{ml}$ plastic cups for one week under stressful conditions followed by one week under non-stressful conditions $(n=218)$. The specific ages of these individuals were unknown. Whole-animal fresh weights were measured before and after each growth period; prior to weighing, individuals were rinsed briefly in distilled water then blotted. Stressful conditions (limited food, low soil moisture) consisted of $20 \mathrm{~g}$ dry culture medium containing autoclaved, composted cow manure: peat moss: $\mathrm{CaCO}_{3}:: 20: 4: 1$, plus distilled water to bring moisture content to 40 per cent; no additional food was provided. Non-stressful conditions (abundant food, high soil moisture) consisted of $20 \mathrm{~g}$ dry culture medium plus distilled water to 70 per cent moisture content; commercially prepared earthworm food (Carolina Biological Supply) was provided ad libitum. These conditions had been shown to be stressful and non-stressful, respectively, in pilot experiments as determined by differences in individual growth. This experimental design is analogous to those studies in which growth is inferred or measured of individuals collected in the field and then remeasured of the same individuals under specific conditions in the laboratory (e.g., Koehn et al., 1988).

Moisture content was monitored daily with an Instamatic ${ }^{\circledR}$ moisture meter; distilled water was added to bring moisture content to the desired level after overnight evaporation. Soil $p \mathrm{H}$, monitored daily with a Brookstone ${ }^{\circledR}$ soil $p \mathrm{H}$ meter, remained at 7 for the duration of the experiment. Ambient temperature was $23-25^{\circ} \mathrm{C}$. Only aclitellate juveniles were used in the experiment; individuals which attained sexual maturity during the course of the experiment, determined by the development of a clitellum, were not considered further. During the experiment, mortality was less than 2 per cent; the final sample size was 169 individuals.

The genotypes of 13 polymorphic loci (ten enzymes) in carbohydrate metabolism were resolved by horizontal starch gel electrophoresis according to Koehn et al. (1976). Approximately $100 \mathrm{mg}$ of the posterior region of each earthworm was homogenized with $100 \mu \mathrm{l}$ grinding buffer $(0.05 \mathrm{M}$ Tris $/ \mathrm{HCl}, 20$ per cent glycerol, $0 \cdot 2$ per cent $\beta$-mercaptoethanol) using a motorized conical teflon tissue homogenizer in a $1.5 \mathrm{ml}$ microcentrifuge tube. Homogenates were centrifuged at $10,000 \mathrm{~g}$ for $20 \mathrm{~min}$ in an Eppendorf model 5415 microcentrifuge; the supernatant was used as the enzyme source. The following enzymes (with E.C. number; locus abbreviation; metabolic function) were resolved: hexokinase (EC 2.7.1.1; Hk1, Hk2; glycolysis), mannose-6-phosphate isomerase (EC 5.3.1.8; Mpi; preglycolysis), L-iditol dehydrogenase (EC 1.1.1.14; Iddh1; preglycolysis), glucose dehydrogenase (EC 1.1.1.47; Glcdh; pentose shunt), phosphoglucomutase (EC 5.4.2.2; Pgm; preglycolysis), glycerol-3-phosphate dehy- 
drogenase (EC 1.1.1.8; Gpdh1, Gpdh2; glycerol metabolism), phosphogluconate dehydrogenase (EC 1.1.1.44; Pgdh; pentose shunt), L-lactate dehydrogenase (EC 1.1.1.27; $L d h 1, L d h 2$; redox balance), strombine dehydrogenase (EC 1.5.1.-; Stdh; redox balance), malate dehydrogenase (EC 1.1.1.37; $M d h$; citric acid cycle).

Electromorphs of various loci were separated using buffer systems described by Selander and Yang (1969). Hk1, Hk2, Mdh, Gpdh1, Gpdh2, and Pgm were resolved on a Tris-borate-EDTA (TBE) buffer system; $P g d h, M p i, L d h 1$, and $L d h 2$ were resolved on a Tris-maleate (TM) buffer system; Stdh, Glcdh, and Iddh1 were resolved on a discontinuous Tris-citrate (Poulik) buffer system. The appropriate cofactor (NADP or NAD) was added to the TBE and TM gels prior to degassing (final concentration: $0.2 \mathrm{mM}$ ) to improve resolution of the dehydrogenases. Activities of $\mathrm{HK}$, IDDH, LDH, MDH, PGM, PGDH were detected according to Shaw and Prasad (1970); activities of GPDH, MPI, GlcDH were detected according to Harris and Hopkinson (1976); activity of StDH was detected according to Dando et al. (1981).

The data were analyzed on an IBM-PC ${ }^{\circledR}$ with a 30 megabyte Flashcard ${ }^{\circledR}$. Statistical programs included the PROC FREQ and PROC GLM procedures from the Statistical Analysis System software for personal computers (SAS Institute, Inc., Cary, NC). Other statistics were from Sokal and Rohlf (1981).

The effect of multilocus heterozygosity on growth rate was tested according to two models. Model A, consistent with traditional approaches to this phenomenon (Zouros et al., 1980; Koehn and Gaffney, 1984), assumed that the effect of individual loci on growth rate was equal and additive among loci. For each locus, individuals were scored 0 if homozygous or 1 if heterozygous. Multilocus heterozygosity was calculated as the sum of these scores over $n$ loci; effect of multilocus heterozygosity was then evaluated by simple linear regression or product-moment correlation. Model $B$ allowed the effects of individual loci to be unequal and non-additive (Bush et al., 1987; Koehn et al., 1988). Individuals were scored as above but the effects of heterozygosity among $n$ loci on growth were evaluated by multiple regression in which loci occurred as independent variables. Each model was evaluated for predicting the effects of heterozygosity on growth measured as initial weight, as weight added under stressful conditions, and as weight added under nonstressful conditions.

\section{RESULTS}

In E. foetida, the average heterozygosity among 13 polymorphic loci in carbohydrate metabolism was 0.397. Of these loci, Iddh $1, L d h 1, L d h 2, S t d h$ showed significant deviations from Hardy-Weinberg equilibrium (table 1 ). In the case of Iddh 1 , $L d h 1$, and $L d h 2$, this deviation represented a heterozygote deficiency; in the case of $S t d h$, this represented a heterozygote excess. The overall heterozygote deficiency $(\mathrm{D}=[\mathrm{Ho}-\mathrm{He}] / \mathrm{He}$; Selander, 1970) was only -0.046 , reflecting a general lack of inbreeding. Linkage disequilibrium was tested among all possible pairs of loci by Pearson's $\chi^{2}$ test for independent association among genotypes. Di-locus genotypes represented by fewer than five individuals were pooled into a single category. Of the 78 possible combinations, only five pairs of loci showed significant deviations from independent association (table 2). Of course at $\alpha=0.05$, approximately 4 significant deviations from independent association are expected on the basis of chance alone; thus the extent of true linkage disequilibrium may be quite small. Nevertheless, three possible linkage groups were identified: Hk1-Pgdh-Iddh1, Ldh1-Ldh2-Stdh, and $G p d h 1-M d h$.

Added weight was a positive function of individual weight prior to a growth period under both stressful and non-stressful conditions; however, the growth trajectories differed significantly between experimental regimes ( $F=$ $41.90 ; \mathrm{df}=1,334 ; P<0.001$; fig. 1$)$. As expected, weight added under non-stressful conditions was significantly greater than weight added under stressful conditions (paired $t=31 \cdot 88 ; \mathrm{df}=168$;

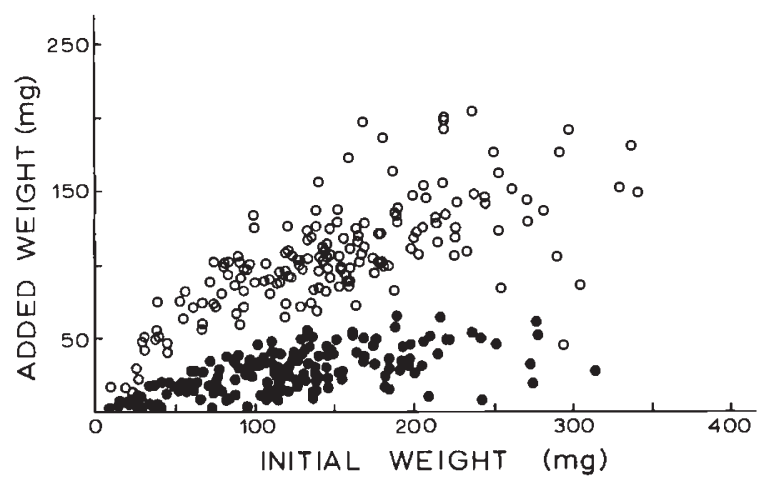

Figure 1 Correlation between added weight and initial weight for both stressful (closed circles; $r=0.592 ; \mathrm{df}=167 ; P<$ 0.001 ) and non-stressful (open circles; $r=0.699$; $\mathrm{df}=167$; $P<0.001)$ growth conditions. Initial weight is defined as the weight of an individual prior to the respective growth period. 
Table 1 Allele frequencies, observed heterozygosity $(H o)$, heterozygote-deficiency $(D)$ for the polymorphic loci of carbohydrate metabolism in Eisenia foetida. Goodness of fit to HardyWeinberg proportions was tested by $\chi^{2}$; genotypes represented by less than five individuals were pooled into an adjacent category (Hedrick, 1985). $n=169$ for each locus

\begin{tabular}{|c|c|c|c|c|c|c|}
\hline Locus & allele & freq & Ho & $D$ & $\chi^{2}(\mathrm{df})$ & $P$ \\
\hline$H k 1$ & $\begin{array}{l}110 \\
105 \\
100\end{array}$ & $\begin{array}{l}0 \cdot 240 \\
0 \cdot 370 \\
0 \cdot 391\end{array}$ & 0.698 & 0.067 & $2 \cdot 986(3)$ & ns \\
\hline$H k 2$ & $\begin{array}{l}110 \\
100\end{array}$ & $\begin{array}{l}0.050 \\
0.950\end{array}$ & 0.089 & -0.066 & nt & \\
\hline$M p i$ & $\begin{array}{l}110 \\
100\end{array}$ & $\begin{array}{l}0.361 \\
0.639\end{array}$ & 0.461 & 0.0004 & $0 \cdot 001(1)$ & ns \\
\hline$I d d h 1$ & $\begin{array}{r}105 \\
100 \\
95 \\
90\end{array}$ & $\begin{array}{l}0.391 \\
0.414 \\
0.098 \\
0.098\end{array}$ & 0.396 & -0.398 & $59 \cdot 048(4)$ & $<0.001$ \\
\hline Glcdh & $\begin{array}{r}110 \\
100 \\
90\end{array}$ & $\begin{array}{l}0 \cdot 192 \\
0 \cdot 509 \\
0 \cdot 299\end{array}$ & 0.562 & -0.085 & $5 \cdot 186(3)$ & ns \\
\hline$P g m$ & $\begin{array}{l}110 \\
100\end{array}$ & $\begin{array}{l}0 \cdot 281 \\
0.719\end{array}$ & 0.420 & 0.040 & $0.018(1)$ & ns \\
\hline$G p d h 1$ & $\begin{array}{r}100 \\
90\end{array}$ & $\begin{array}{l}0.917 \\
0.083\end{array}$ & $0 \cdot 166$ & 0.088 & nt & \\
\hline$G p d h 2$ & $\begin{array}{l}110 \\
100\end{array}$ & $\begin{array}{l}0.459 \\
0.541\end{array}$ & 0.462 & -0.071 & $1 \cdot 149(1)$ & ns \\
\hline$P g d h$ & $\begin{array}{l}110 \\
100\end{array}$ & $\begin{array}{l}0.240 \\
0.760\end{array}$ & 0.396 & 0.087 & $1 \cdot 305(1)$ & ns \\
\hline$L d h 1$ & $\begin{array}{l}110 \\
100\end{array}$ & $\begin{array}{l}0.275 \\
0.725\end{array}$ & 0.290 & -0.273 & $12 \cdot 606(1)$ & $<0.001$ \\
\hline$L d h 2$ & $\begin{array}{r}110 \\
100 \\
90\end{array}$ & $\begin{array}{l}0.062 \\
0.754 \\
0.183\end{array}$ & 0.320 & -0.185 & $6 \cdot 781(2)$ & $<0.05$ \\
\hline Stdh & $\begin{array}{r}100 \\
90\end{array}$ & $\begin{array}{l}0.784 \\
0.216\end{array}$ & 0.402 & $0 \cdot 188$ & $4.921(1)$ & $<0.05$ \\
\hline$M d h$ & $\begin{array}{r}100 \\
90\end{array}$ & $\begin{array}{l}0.518 \\
0.482\end{array}$ & 0.503 & 0.007 & $0.161(1)$ & ns \\
\hline
\end{tabular}

$\mathrm{nt}=$ Hardy-Weinberg not tested due to insufficient sample size. ns $=$ not significant.

Table 2 Pairs of loci whose genotypes are not associated independently, tested by the Pearson $\chi^{2}(a=0.05)$. Dilocus genotypes represented by less than five individuals were pooled into a single category

\begin{tabular}{crrl}
\hline Di-locus pair & $\chi^{2}$ & df & $P$ \\
\hline Hk1, Pgdh & 39.594 & 10 & $<0.001$ \\
Pgdh, Iddh1 & 24.265 & 11 & $<0.05$ \\
Ldh1, Ldh2 & 17.247 & 9 & $<0.05$ \\
Ldh2, Stdh & 26.410 & 4 & $<0.001$ \\
Gpdh1,Mdh & 9.823 & 2 & $<0.01$ \\
\hline
\end{tabular}

$P<0.001$; table 3 ); yet the coefficient of variation for added weight was greater under the latter condition.

Contrary to the results of previous studies, multilocus heterozygosity (heterozygosity summed across loci; model A) was not significantly correlated with initial weight $(r=0.045 ; \mathrm{df}=167 ; \mathrm{NS})$; with weight added under stressful conditions $(r=$ $0.050 ; \mathrm{df}=167 ; \mathrm{NS}$ ) or with weight added under non-stressful conditions $(r=-0.058 ; \mathrm{df}=167$; NS; table 4). A significant correlation was detected only between heterozygosity among loci treated separ- 
Table 3 Effect of supplemental food and moisture content on added fresh weight $(\mathrm{mg})$ in Eisenia foetida. Where appropriate, data are presented as means (standard error). $n=169$

\begin{tabular}{|c|c|c|}
\hline & $\begin{array}{l}\text { Week } 1 \\
\text { (stressful) }\end{array}$ & $\begin{array}{l}\text { Week } 2 \\
\text { (non-stressful) }\end{array}$ \\
\hline Supplemental food & none & ad libitum \\
\hline Soil moisture content & $30-40 \%$ & $60-70 \%$ \\
\hline Added fresh weight & $27 \cdot 6(1 \cdot 13)$ & $105 \cdot 4(2 \cdot 86)$ \\
\hline Coefficient of variation & $53.4(3.64)$ & $35 \cdot 3(2 \cdot 14)$ \\
\hline
\end{tabular}

Table 4 Coefficients of determination $\left(r^{2}\right.$ or $R^{2}$ ) between two models predicting the effects of heterozygosity on 3 measures of growth. Model A assumes individual loci contribute similarly to growth rate and that effects are additive; model B assumes individual loci contribute differentially and that effects are not additive. Significance tested by ANOVA; model $A$, linear regression, $\mathrm{df}=1,167$; model $\mathrm{B}$, multiple regression, $\mathrm{df}=13,155$

\begin{tabular}{lll}
\hline Dependent variable & Model A & Model B \\
\hline Initial weight & $0 \cdot 002$ & $0 \cdot 082$ \\
& $F=0 \cdot 342, \mathrm{~ns}$ & $F=1 \cdot 071, \mathrm{~ns}$ \\
Weight added & $0 \cdot 002$ & $0 \cdot 164$ \\
$\quad$ (stressful env.) & $F=0 \cdot 416, \mathrm{~ns}$ & $F=2 \cdot 344, P<0.01$ \\
Weight added & $0 \cdot 003$ & $0 \cdot 118$ \\
$\quad$ (non-stressful env.) & $F=0 \cdot 350, \mathrm{~ns}$ & $F=1 \cdot 600, \mathrm{~ns}$ \\
\hline
\end{tabular}

ately (model B) and weight added under stressful conditions $(R=0 \cdot 405 ; F=2 \cdot 344 ; \mathrm{df}=13,155 ; P<$ 0.01 ; table 4 ). Indeed heterozygosity among loci treated separately explained more than 15 per cent of the variation in added weight under these conditions but showed no significant effect on either initial weight $(R=0 \cdot 287 ; F=1 \cdot 071 ; \mathrm{df}=13,155$; NS) or weight added under non-stressful conditions $(R=0.344 ; F=1 \cdot 600 ; \mathrm{df}=13,155 ; \mathrm{NS})$. Clearly these data support a model which assumes differential, possibly non-additive effects among loci. Moreover, this genetic effect is better visualized under conditions of environmental stress.

The contribution of heterozygosity at single loci toward explaining variation in growth under stressful conditions was evaluated by ranking the partial sums of squares from a multiple regression while considering the sign of the partial regression coefficients (table 5). Heterozygosity at two loci, $G p d h 2$ and $H k 2$, showed significant individual positive effects on added weight; heterozygosity at one locus, Iddh1, showed a significant individual negative effect. In all, seven loci contributed positively; whereas, six loci contributed negatively, based simply on the sign of the partial regression
Table 5 Rank of single loci contributing to the relationship between added weight and heterozygosity among loci for Eisenia foetida under stressful conditions (limited food, low moisture). Effects of initial weight have been taken into account by multiple regression

\begin{tabular}{|c|c|c|c|c|c|}
\hline Locus & $S S$ & $\begin{array}{l}\text { Partial } \\
\text { regression } \\
\text { coefficient }\end{array}$ & $r^{2}$ & $\begin{array}{l}F \\
(1,154)\end{array}$ & $P$ \\
\hline$G p d h 2$ & 0.00081 & 0.0046 & 0.025 & $6 \cdot 327$ & $<0.05$ \\
\hline$H k 2$ & $0 \cdot 00060$ & 0.0070 & 0.019 & $4 \cdot 738$ & $<0.05$ \\
\hline$L d h 1$ & 0.00030 & 0.0031 & 0.009 & $2 \cdot 328$ & ns \\
\hline$P g m$ & 0.00019 & 0.0023 & 0.006 & 1.459 & ns \\
\hline$M d h$ & 0.00016 & 0.0021 & 0.005 & $1 \cdot 285$ & ns \\
\hline$L d h 2$ & 0.00006 & 0.0014 & 0.002 & 0.459 & ns \\
\hline Glcdh & $0 \cdot 00002$ & 0.0006 & 0.001 & $0 \cdot 120$ & ns \\
\hline$H k 1$ & 0.00011 & -0.0018 & 0.003 & 0.869 & ns \\
\hline$G p d h 1$ & 0.00015 & -0.0026 & 0.005 & $1 \cdot 207$ & ns \\
\hline$M p i$ & 0.00024 & -0.0025 & 0.008 & $1 \cdot 888$ & ns \\
\hline Pgdh & 0.00028 & -0.0027 & 0.009 & $2 \cdot 190$ & ns \\
\hline Stdh & 0.00037 & -0.0034 & 0.012 & 2.919 & ns \\
\hline$I d d h 1$ & 0.00057 & -0.0040 & 0.018 & 4.492 & $<0.05$ \\
\hline
\end{tabular}

coefficients. Thus sampling smaller subsets of polymorphic loci, as is typical of most studies, may reveal starkly different results depending on the loci chosen. For example, if loci which showed only a positive effect were arbitrarily sampled, the equal and additive model would not be refuted and multilocus heterozygosity would explain about five per cent of the variation in growth (fig. 2). Interestingly, if negatively-contributing loci were arbitrarily sampled, multilocus homozygosity would explain about four per cent of the variation in growth. In contrast to the study of Koehn et al (1988), it is not possible to associate single-locus contributions to growth in E. foetida with obvious metabolic functions in carbohydrate metabolism. This is particularly evident for enzymes such as HK and GPDH both of which are encoded by two loci: one contributing positively, the other contributing negatively.

The existence of both positively and negatively contributing loci suggests that the effects of heterozygosity may not always be additive among loci, possibly due to interactions among loci: physical (linkage), functional (epistasis) or both. Differences in growth among genotypes of single loci were detected only for $G p d h 2(F=4.77$; df $=$ $3,165 ; P<0.01)$ and $M d h(F=3.68 ; \mathrm{df}=2,166$; $P<0.05)$; yet, differences in growth among trilocus genotypes were detected for the $L d h 1-L d h 2$ Stdh linkage group (ANOVA; $F=1.75$; df $=25$, $141 ; P<0.025)$, suggesting that some interactions may exist. Genotypic interactions between loci were detected in only three pairs of loci: $H k 2, M p i$ $(F=4 \cdot 16 ; \mathrm{df}=2,162 ; P<0.025) ; G p d h 2, \quad I d d h 1$ 


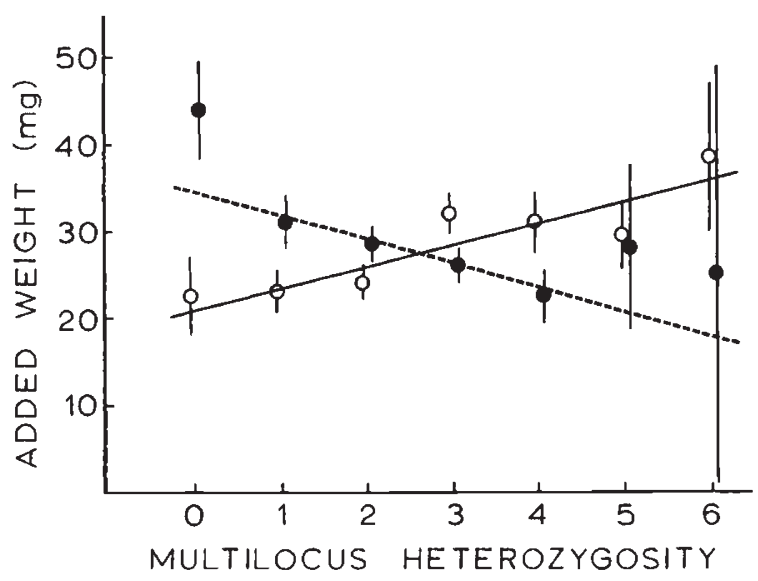

Figure 2 Possible relationships between individual multilocus heterozygosity and growth under stressful conditions if subsets of polymorphic loci are arbitrarily chosen for study. Solid line, open circles: multilocus heterozygosity was calculated from positively contributing loci $(F=9.88$; $\mathrm{df}=$ $1,167 ; P<0.005)$. Dashed line, closed circles: multi-locus heterozygosity was calculated from negatively contributing loci $(F=7.15 ; \mathrm{df}=1,167 ; P<0.01)$. Data are presented as means \pm standard error.

$(F=1 \cdot 76 ; \mathrm{df}=15,141 ; P<0.05) ; \operatorname{Mpi}, \operatorname{Pgm}(F=$ $2.64 ; \mathrm{df}=4,159 ; P<0.05)$ and no higher order genotypic interactions were detected within either of the presumed tri-locus linkage groups. Of course, the small sample size of individuals in this experiment militates against detecting any such interactions.

\section{DISCUSSION}

Eisenia foetida has been described as an autofecundable amphimictic hermaphrodite, in which parthenogenesis has not been demonstrated (Hartenstein et al., 1980). Though limited selffertilization may occur, E. foetida generally reproduces sexually, (Reynolds et al., 1973; Hartenstein et al., 1979, 1980), producing two cocoons per mating with 1-5 viable offspring per cocoon (Hartenstein et al., 1979). Individuals may reach sexual maturity in 4-6 weeks, indicated by the presence of a clitellum, and may produce as many as five cocoons per week during peak reproductive output (Hartenstein et al., 1979). When self-fertilization does occur, cocoon viability is only $\frac{1}{4}$ that resulting from amphimictic parents (Hartenstein et al., 1979, 1980). It is not known to what extent, if at all, self-fertilization occurs in large cultures.

In the present experiment, juvenile $E$. foetida were presumably produced randomly by sexual reproduction among individuals of a large effective parental stock. Yet the possibility that self-fertilization might occur or that the effective number of sexually-reproducing parents might be small due to non-random mating would suggest that substantial amounts of inbreeding could exist. Limited parentage has been shown to militate against observing a heterozygosity-growth relationship (Gaffney and Scott, 1984). Substantial inbreeding is detectable by any one of several analyses; yet in the present experiment, little evidence of inbreeding was detected. The average heterozygote deficiency $(D)$ among 13 loci was small, $-0 \cdot 046$. Of 78 possible pairwise combinations of loci, the genotypes of only five pairs of loci were associated non-randomly, suggesting a general lack of linkage disequilibrium which is expected under substantial inbreeding. Indeed, E. foetida, a diploid species, has only 11 pairs of chromosomes (Muldal, 1952; Omodeo, 1952), thus some linkage among 13 loci might be expected even in the absence of extensive inbreeding. For the few loci which have been resolved in common ( $L d h 2, M d h, P g m$ ) between this study and one which sampled a natural, endemic population of E. foetida from Italy (Robotti, 1982), the observed heterozygosity and number of alleles per locus were similar.

Only the significant heterozygote deficiencies at three loci ( $I d d h 1, L d h 1$, and $L d h 2$ ) suggest some peculiarity of population structure. Jaenike (1982) has suggested that $E$. foetida is made up of two biological species: a striped morph, E. foetida foetida, and an unstriped morph, E. foetida andrei; crosses between these morphs produce sterile hybrids. Thus mixture of two species each with different allele frequencies at these loci could produce the heterozygote deficiencies observed.

However, the commercial population sampled in the present experiment probably represents only one species. Morphologically, it was not possible to separate the sample into two subsets on the basis of presence or absence of stripes. Pgm, the only locus resolved in common between the study of Jaenike (1982) and the present study, is polymorphic in both morphs of E. foetida, with two common alleles per morph. However, no alleles are shared by the two morphs (Jaenike, 1982). Thus a mixture of E. f. foetida and E. f. andrei should reveal four alleles at the $\mathrm{Pgm}$ locus plus a striking heterozygote deficiency. Yet in the present study, only two alleles were detected; the frequencies of these alleles were in Hardy-Weinberg equilibrium and were similar to those of the unstriped morph reported by Jaenike (1982). Further, glucose-6phosphate isomerase ( $\mathrm{Gpi}$ ) is polymorphic in the 
striped morph but monomorphic in the unstriped morph (Jaenike, 1982). Initial surveys of the genetic properties of glycolytic enzymes revealed Gpi to be monomorphic in the present experiment. Thus evidence suggests that the commercial population of $E$. foetida is a mono-culture of $E$. foetida andrei and adequately represents the species as a whole. The initial assumption of a large, panmictic population is probably valid.

Only weight added under stressful conditions was significantly correlated with heterozygosity among loci treated separately $(R=0.405 ; \mathrm{df}=167$; $P<0.01$ ); no correlations existed for any measure of growth with multilocus heterozygosity treated cumulatively. Possible reasons for this, summarized by Koehn et al. (1988), include (1) strong inbreeding or non-random mating, (2) limited parentage, (3) genotype-dependent mortality, (4) mixed age classes in the population, (5) lack of sufficient environmental stress, or (6) inappropriate choice of loci due to differential effects. Thus, correlations would not be expected between heterozygosity, however treated, and initial weight as a consequence of the multiple age classes represented in the sample. In subsequent measures of growth, initial weight, and presumably age, was taken into account. Further, it was not surprising to find a lack of a correlation between heterozygosity and growth under non-stressful conditions because these duplicated conditions known to promote optimal growth in this species: temperatures of $20-29^{\circ} \mathrm{C}$, soil moisture of $70-85$ per cent, and pH 6-8 (Kaplan et al., 1980).

The most likely explanation for the lack of a traditional multilocus heterozygosity-growth relationship remains the differential contribution to growth of heterozygosity among loci. Indeed the predictive value of heterozygosity among loci treated separately is much greater than that of traditional multilocus heterozygosity for all three measures of growth. The improved fit generated by models which treat loci separately was originally proposed by Smouse (1986) and applied by Bush et al. (1987) to the data of Ledig et al. (1983) for the pitch pine Pinus rigida. Bush et al. (1987) showed that models which treat loci separately may explain ten times more variation in growth than that explained by models which treat loci cumulatively. An important assumption of these "separate-locus" models is that single locus effects are indeed different from one another, an assumption demonstrated for E. foetida. In addition, the present results suggest that single locus effects may not be additive, since several loci apparently contributed negatively to growth.
What accounts for the rank of loci contributing to growth? Koehn et al. (1988) showed that degree of heterozygosity per locus did not predict the magnitude of the individual locus effect. Similarly in the present experiment, the rank of loci contributing to growth was not correlated with either rank of heterozygote deficiency (Spearman $r_{s}=$ $-0 \cdot 308$; NS) or with rank of observed heterozygosity per locus (Spearman $r_{s}=-0.037$; NS). Koehn et al. (1988) further demonstrated that loci encoding enzymes of protein catabolism, preglycolysis, or glycolysis had significant effects on growth; whereas, loci encoding enzymes of other metabolic functions, (e.g., pentose shunt, digestion, redox balance, or detoxification) did not. The failure to include protein catabolic enzymes in the present study may be responsible, in part, for the lack of a traditional multilocus heterozygosity-growth relationship. Nevertheless, heterozygosity, or the lack of it, among enzymes of carbohydrate metabolism in E. foetida clearly has a highly significant effect on growth. Still it is not possible to subdivide loci into convenient metabolic pathways within carbohydrate metabolism on the basis of their contribution to growth.

Although available evidence certainly suggests that individual loci contribute differently to growth, these are some of the few results which suggest that heterozygosity at several loci may contribute negatively to growth; previously most studies have shown heterozygosity to contribute either positively or neutrally (but see Ledig et al., 1983; Diehl and Koehn, 1985). Consequently the nature of the multilocus heterozygosity-growth relationship is probably more complex than previously recognized and depends greatly on the loci chosen for study and possibly on the environment to which individuals are subjected.

There seem to be three possible reasons for heterozygotes contributing negatively toward growth; heterozygote inferiority, linkage disequilibria, or strong selection at the particular locus. These possibilities cannot be fully evaluated in the present experiment because of the limited sample size; however some comment is pertinent.

Of these possibilities, heterozygote inferiority (underdominance) is the most difficult case to support. Heterozygote inferiority is poorly studied, but it may theoretically occur if two homozygotes are adapted to two different resources, whereas the heterozygote is adapted to neither (Futuyma, 1986). Extending this situation to the current experiment is unwarranted at present. Interestingly, if heterozygote inferiority were shown to account for the negative effect on growth, any 
postulated mechanism would virtually require a causal relationship between the implicated loci and growth. Accumulation of advantageous recessive alleles among inbred individuals could not be invoked to account for a homozygosity-growth phenomenon in the same way that linked deleterious recessive alleles are occasionally invoked to explain the heterozygosity-growth phenomenon (see Mitton and Grant, 1984).

If certain loci sampled in the present experiment were neutral in their effect on growth but also were in strong linkage disequilibria with positively contributing loci, then one could construct specific chromosomal patterns which would produce the association of positive effects and negative effects observed. If this were the case, the specific patterns described in the present experiment should not be duplicated if the experiment were repeated with another population or even another subset of this population. However at present, this argument remains difficult to sustain in the absence of substantial linkage disequilibrium, especially considering the great potential for its existence.

Theoretically, strong selection for a particular locus would produce homozygotes whose fitness is greater, on the average, than that of heterozygotes when at equilibrium under a balance between mutation and stabilizing selection (Slatkin, 1987). When selection is weak, on the other hand, the fitness of heterozygotes is predicted to be greater than that of homozygotes as a consequence of the narrower variance of the trait measured in heterozygotes (Slatkin, 1987). Consequently, strongly selected loci might produce a negative heterozygosity-growth relationship superimposed on the positive relationship generated among weakly selected loci. However, these predictive patterns cannot be invoked to explain the observed patterns until the phenotypes of negatively contributing loci are shown to be strongly selected in response to specific environmental conditions, a situation awaiting further study.

Acknowledgements I would like to thank R. K. Koehn, P. Gaffney, and D. Wise for useful comments on this paper.

\section{REFERENCES}

BUSH, R. M., SMOUSE, P. E. AND LEDIG, F. T. 1987. The fitness consequences of multiple-locus heterozygosity: the relationship between heterozygosity and growth rate in pitch pine (Pinus rigida Mill.). Evolution, 41, 787-798.
DANDO, P. R., STOREY, K. B. HOCHACHKA, P. W. AND STOREY, J. M. 1981. Multiple dehydrogenases in marine molluscs: electrophoretic analysis of alanopine dehydrogenase, strombine dehydrogenase, octopine dehydrogenase and lactate dehydrogenase. Mar. Biol. Lettrs., 2, 249-257.

DASTOLLI, F. R. 1964. The intermediary carbohydrate metabolism of Lumbricus terrestris. J. Cell. Comp. Physiol., 64, 465-472.

DIEHL, W. J. AND KOEHN, R. K. 1985. Multiple-locus heterozygosity, mortality, and growth in a cohort of Mytilus edulis. Mar. Biol, 88, 265-271.

DIEHL, W. J., GAFFNEY, P. M. AND KOEHN, R. K. 1986. Physiological and genetic aspects of growth in the mussel Mytilus edulis. I. Oxygen consumption, growth, and weight loss. Physiol. Zool., 59, 201-211.

FUTUYMA, D. J. 1986. Evolutionary Biology. Sinauer Associates, Inc., Sunderland, MA.

GAFFNEY, P. M. AND SCOTT, T. 1984. Genetic heterozygosity and production traits in natural and hatchery populations of bivalves. Aquacult., 42, 289-302.

HARRIS, H. AND HOPKINSON, D. A. 1976. Handbook of Enzyme Electrophoresis in Human Genetics. North Holland Publishing Co., Amsterdam.

HARTENSTEIN, R., NEUHAUSER, E. F. AND KAPLAN, D. L. 1979. Reproductive potential of the earthworm Eisenia foetida. Oecologia, 43, 329-340.

HARTENSTEIN, R., NEUHAUSER, E. F. AND EASTON, E. G. 1980. Growth and fecundity of $\mathrm{F}_{2}$ Eisenia foetida derived from $\mathrm{F}_{1} \mathrm{~s}$, both reared in isolation from birth. Megadrilogica, 3, 185-187.

HAWkins, A. J. S., BAYNe, B. L. AND DAY, A. J. 1986. Protein turnover, physiological energetics and heterozygosity in the blue mussel, Mytilus edulis: the basis of variable agespecific growth. Proc. R. Soc. Lond. B., 229, 161-176.

HEDRICK, P. W. 1985. Genetics of Populations. Jones and Bartlett Publishers, Inc., Boston.

JAENIKE, J. 1982. "Eisenia foetida" is two biological species. Megadrilogica, 4, 6-8.

KAPLAN, D. L:, HARTENSTEIN, R., NEUHAUSER, E. F. AND MALECKI, M. R. 1980. Physicochemical requirements in the environment of the earthworm Eisenia foetida. Soil Biol. Biochem., 12, 347-352.

KOEHN, R. K., MILKMAN, R. AND MitToN, J. B. 1976. Population genetics of marine pelecypods. IV. Selection, migration and genetic differentiation in the blue mussel Mytilus edulis. Evolution, 30, 2-32.

KOEHN, R. K. AND SHUMWAY, S. E. 1982. Agenetic/physiological explanation for differential growth rate among individuals of the American oyster, Crassostrea virginica (Gmelin). Mar. Biol. Lettrs., 3, 35-42.

KOEHN, R. K. AND GAFFNEY, P. M. 1984. Genetic heterozygosity and growth rate in Mytilus edulis. Mar. Biol, , 82, 1-7.

KOEHN, R. K., DIEHL, W. J. AND SCOTT, T. M. 1988. The differential contribution by individual enzymes of glycolysis and protein catabolism to the relationship between heterozygosity and growth rate in the coot clam, Mulinia lateralis. Genetics, 118, 121-130.

LEDIG, F. T., GURIES, R. P. AND BONEFELD, B. A. 1983. The relation of growth to heterozygosity in pitch pine. Evolution, 37, 1227-1238.

LEE, K. E. 1985. Earthworms their Ecology and Relationships with Soils and Land Use. Academic Press, Sydney.

MITTON, J. B. AND GRANT, M. C. 1984. Associations among protein heterozygosity, growth rate, and developmental homeostasis. Ann. Rev. Ecol. Syst., 15, 479-499.

MULDAL, S. 1952. The chromosomes of the earthworms. I. The evolution of polyploidy. Heredity, 6, 56-76. 
OMODEO, P. 1952. Cariologia dei Lumbricidae. Caryologia, 4, 173-275.

REYNOLDS, J. W., CLEBSCH, E. E. C. AND REYNOLDS, W. M. 1974. Contributions to North American earthworms (Oligochaeta) No. 13 The earthworms of Tennessee (Oligochaeta) I. Lumbricidae. Bull. Tall Timbers Res. Stn., $17,1-133$.

ROBOTTI, C. A. 1982. Biochemical polymorphism of earthworms. 4. Enzymes of Eisenia fetida andrei Bouche (Annelida Oligochaeta). Monitore zool. ital. (N.S.), 16, 341-344.

RODHOUSE, P. G. AND GAFFNEY, P. M. 1984. Effect of heterozygosity on metabolism during starvation in the American oyster Crassostrea virginica. Mar. Biol., 80, 179-187.

SELANDER, R. K. 1970. Behavior and genetic variation in natural populations. Am. Zool., 10, 53-66.

SELANDER, R. K. AND YANG, S. Y. 1969. Protein polymorphism and genic heterozygosity in a wild population of the house mouse (Mus musculus). Genetics, 63, 653-667.
SHAW, C. R. AND PRASAD, R. 1970. Starch gel electrophoresis of enzymes-a compilation of recipes. Biochem. Genet., 4 , 297-320.

SLATKIN, M. 1987. Heritable variation and heterozygosity under a balance between mutations and stabilizing selection. Genet. Res., Camb., 50, 53-62.

SMOUSE, P. E. 1986. The fitness consequences of multiple-locus heterozygosity under the multiplicative overdominance and inbreeding depression models. Evolution, 40, 946-957.

SOKAL, R. R. AND ROHLF, F. J. 1981. Biometry, W. H. Freeman and Co., San Francisco.

ZOUROS, E., SINGH, S. M. AND MILES, H. E. 1980. Growth rate in oysters: an overdominant phenotype and its possible explanations. Evolution, 34, 856-867.

ZOUROS, E. AND FOLTZ, D. w. 1987. The use of allelic isozyme variation for the study of heterosis. Isozymes: Current Topics Biol. Med. Res., 13, 1-59. 